\title{
SOME INDICATIONS FOR HORMONE THERAPY, AND ITS RATIONALE.
}

\author{
By S. LEVY SIMPSON, M.A., M.D. (Camb.), M.R.C.P. (Lond.)
}

(Physician to Willesden General Hospital, Assistant Physician, Princess Louise Kensington Hospital for Children. Formerly Research worker in Endocrinology, Lister Institute.)

As a departure from usual practice, I shall consider the various indications for organotherapy under the classification of hormones rather than diseases. Hormone therapy is always substitution therapy, but it may be (a) direct e.g. oestrin, or (b) indirect e.g. gonadotropic, by which another endocrine gland e.g. the ovary, is stimulated to resume activity. Even in the latter case, it is still substitution therapy (of the pituitary), but it would appear that if some extraneous help can be given to the endocrine mechanism, autonomous activity may be resumed after therapy has ceased. This' is very much to be hoped for, since, with the "tropic" hormones, continuous therapy may be ineffectual, owing to the production in the body of anti-hormonic substances, which render the "tropic" hormones inactive. Intermittent therapy may overcome this difficulty to some extent.

This anti-hormonic mechanism is not limited to the "tropic" hormones, and may affect others e.g. parathyroid hormone. In general, however, the pituitary "tropic" hormones are of a complex chemical formula likely to produce antihormonic substances, while the direct hormones of other endocrine glands e.g. œstrone, testosterone, have a much simpler chemical composition, and yield pure crystals.

\section{GONADOTROPIC HORMONES.}

In order to consider the therapeutic indications, it is permissible to divide these into A. and B. A. acts on the germ cell, producing follicle ripening in the ovary, and spermatozoal development in the testes, whereas B. acts on the connective tissue elements, producing luteinization and interstitial cell development in the ovary, and interstitial cell hyperplasia in the testes. Experimental evidence of a complete differentiation into A. and B. has not been accepted, but there is little doubt that different materials and methods of extraction yield a preponderance of $\mathrm{A}$. or $\mathrm{B}$. and that in some preparations, A. may be entirely, or almost entirely, lacking. A. will produce follicle growth in the ovary of a monkey or a hypophysectomized animal, whereas B. has only a slight action on the theca cells, and produces some hyalinization of the follicle. It is important to remember that B. has apparently no true luteinizing effect on the ovary of monkey or woman, although, as we shall see, its effect on menorrhagia might be explained as luteinization, if such occurred. A preponderance of A. occurs in menopausal urine, human castrate urine, and the serum of pregnant mares. A preponderance of B. occurs in human pregnancy urine. The pituitary yields both A. and B.

Commercial preparations, for the most part, are obtained from human pregnancy urines e.g. Pregnyl, Gonan, Antuitrin S., etc., and must be regarded as mainly B. An alleged follicle stimulating hormone (A), Antostab, is obtained from the serum of pregnant mares. The preparations are usually in powder form, containing Ioo or 500 units, to be dissolved in I c.cm. of a given solvent. If already in solution, the latter should be kept in the refrigerator, to prevent some loss of potency. 


\section{GONADOTROPIC B. (most preparations).}

\section{Menorrhagia.}

B. is especially useful in the menorrhagia of puberty, and at the menopause, but may be of value at any age. It is important to exclude gynæcological complications, such as carcinoma, fibroids, polypi, incomplete abortions and metritis.

At the menopause, radiation sterilization is an alternative, but $\mathrm{I}$ have seen climacteric menorrhagia respond to gonadotropic B., and after six weeks therapy, normal menstruation has been established for a further I8 months, after which a gradual, quiet menopause has ensued.

A hundred units twice weekly is usually adequate therapy, but, if necessary, further injections should be given at the time of bleedings.

With puberty menorrhagia, normal menstruation may result after some weeks, and therapy be discontinued.

\section{Undescended testis.}

Unless there is evidence of infantilism, therapy should be postponed until the age of I4, since spontaneous descent of the testis may occur at puberty, and usually no damage to a retained testicle occurs before adolescence. If the testis is ectopic, and therefore mechanically incapable of descending in the canal, surgical aid is essential.

Treatment consists of 500 units given intramuscularly twice weekly for 6 to I2 weeks. Maturation of the interstitial cells result, and may be evidenced by the growth of secondary sexual characteristics. The testis grows in size, and gradually? descends into the scrotum, which also enlarges to receive it. Failure will occur in some 30 or 40 per cent. of cases, but even when surgical aid is necessary, the previous and subsequent endocrine therapy will facilitate the mechanical procedure, and tend to prevent relapse.

\section{Hypogonadism.}

This may be part of infantilism, or of Fröhlich's syndrome, or may have followed mumps, etc. Injections of $B$. will often produce sexual maturity.

\section{GONADOTROPIC A.}

\section{Amenorrhœa.}

Primary amenorrhœe is somewhat refractory to therapy, but secondary amenorrhœa may respond very well. The condition may be of indeterminate origin, but may be due to obvious hypogonadism, as part of an endocrine syndrome. Since follicle stimulation is desirable, quite apart from the fact that the follicles produce ostrin, A. therapy is indicated. Nevertheless, it must be admitted that, in spite of the absence of rationale, $B$. may be apparently effective. Normal menstruation may persist after cessation of therapy.

\section{Aspermia.}

When the patient has normal coitus, but the ejaculated fluid is seen to contain few or no healthy spermatozoa, A. is indicated. B. may abolish impotence, and hypogonadism, but it does not restore spermatogenesis. 


\section{CESTRIN.}

The term $C$ strin is applied to the group of which the pure substances cestrone and oestradiol are the typical representatives, and in the following section the word œstrin will be used for convenience, and the dosage given in international units. The word oestrin was used because the substances produce oestrus, but all are chemically related to the sterols.

Estrone is the ketohydroxy compound found in urine. Estradiol is the dihydroxy compound secreted by the Graafian follicle, and is much more active than œstrone. Commercially, œstrone is obtained from pregnancy urine, converted by a chemical process to œstradiol, and the latter subsequently converted into the ester œstradiol benzoate, which has a slower absorption rate, and a more sustained action owing to the gradual setting free of the oestradiol in the organism. It is sold in ampoules of oily solution under various trade names e.g. Dimenformon, Estroform, Progynon, etc. Vaginal suppositories are also used, and are apparently entirely absorbed. Tablets may be taken by mouth, and are effective to the extent of one tenth of the dosage administered.

\section{Climacteric symptoms.}

The climacteric is associated with a cessation of ovarian activity, which would appear to be an autonomous exhaustion, and not secondary to pituitary failure, since there are many indications of pituitary hyperactivity. In fact, the latter is best explained as a result of the ovarian failure, being comparable to that which follows castration. There is a considerable decline in the amount of ostrin in the urine, and an increase in the amount of gonadotropic hormone. In view of the latter, it is illogical to attempt therapy with gonadotropic hormones, as the ovary has presumably ceased to be capable of responding to such stimuli. (This might not apply to the pre-menopausal phase of menorrhagia.)

The nervous and vaso-motor symptoms of the menopause are probably due to the pituitary overactivity rather than to the primary ostrin deficiency, since they may be abolished by pituitary radiation, or by testosterone propionate, which inhibits pituitary activity. The abolition of these symptoms by ostrin is therefore probably an indirect effect on the pituitary. Although climacteric hypertension and diabetes may also be due to pituitary overactivity, œstrin will not abolish them, but may produce appreciable amelioration.

The local manifestations of the late climacteric, e.g. pruritus vulvæ, kraurosis vulvæ, leucoplakia vulvæ, senile vaginitis, and dyspareunia from local atrophy, are directly due to ostrin deficiency, and are not helped in any way by pituitary radiation, or testosterone propionate. They will, however, readily respond to œstrin therapy.

The dosage of œstrin varies with individuals. In some patients, a daily tablet of $I, 000$ units is adequate, while in others 50,000 units given intramuscularly twice weekly may be called for. The determination of dosage may be left as a clinical problem, based upon the effect on symptomatology. More scientific criteria are (a) the disappearance of an excess of gonadotropic hormone from the urine, $(b)$ the change of the vaginal smear from a leucocytic one to an œstrous one, with the diminution or disappearance of leucocytes, and the appearance of flattened cornified epithelial cells.

In my experience, it is always possible to abolish vasomotor symptoms, and to improve considerably the local symptoms except in the late stages. There are two possible disadvantages of œstrin therapy: $(a)$ the resulting hyperplasia of the 
uterus may produce backache, and uterine colic, and may result in uterine hæmorrhage during therapy, or after cessation of therapy. The latter may be avoided by a gradual reduction of dosage, instead of a sudden cessation. Bleeding during therapy is due to the fact that the dosage is a so-called threshold dose for the particular patient, and may be obviated either by an increase or a decrease of dosage. The uterine pains may be only initial and transitory, but their persistence calls for a gradual decrease of dosage. If a severe hæmorrhage is occurring during or after cestrin therapy, it should be controlled by the injection of 2 units of progesterone, repeated if necessary. I have also found that a large injection of œstrin will cause the hæmorrhage to cease, and this may be of some theoretical as well as practical interest.

The breasts may enlarge, and become tense and painful. Usually these symptoms are transitory, and occur at the beginning of the course of injections. If they persist, the dose of œstrin should be reduced. The possibility of malignant change resulting is remote, but it is perhaps less unlikely that a cystic mastitis would result from therapy with very large doses over a period of years. Usually therapy can be discontinued, or minimized, after some months. Zuckerman's monkeys shewed no pathological changes in the breasts after a year's therapy, but, nevertheless, in view of the above considerations, I advocate the use of the minimal dose of ostrin that will adequately control the symptoms.

\section{After bilateral ovariectomy.}

The alleged injurious effects of this operation appear to have an exaggerated importance in the minds of doctors, and the lay public. In my experience, when the operation is carried out after full sexual development, and before the age of 40 , the symptomatic effects may be negligible. There is rarely any loss of sexual libido, and there may even be an increase. Coitus is usually completely satisfactory. Gain in weight is often only slight, and hypertension rarely considerable. Vasomotor symptoms do, however, occur, but may not be sufficiently severe to call for therapy. Where indicated, however, œstrin therapy will abolish all symptoms.

The fact that a bilateral ovariectomised woman may, nevertheless, at about the age of 45 , experience the various symptoms of the climacteric, suggests that there is another factor, apart from the cessation of ovarian activity, which determines pituitary hyperactivity at this period.

\section{Amenorrhœa.}

Even in primary amenorrhœa, œstrin, in doses of 200,000 units weekly for three weeks, followed by progestin, 5 rabbit units, will produce a menstrual period, but this can only be made to recur by continuing the therapy.

In secondary amenorrhœa, much smaller doses of œstrin, given for three weeks, and then stopped for one week, may re-establish menstruation (without progestin) and this may continue after the cessation of therapy.

It may, however, be necessary to use gonadotropic hormone (see above), but where the uterus is infantile, preliminary therapy with œstrin is often advisable.

\section{Vulvo-vaginitis of children.}

Estrin tablets, Io,000 units each, daily, will usually clear the condition in four to six weeks, the only local treatment being a daily bath or external lavage 
with mild boracic lotions. The œstrin acts by producing cornification, and shedding the infected epithelium.

It may be efficacious in cases that have proved intractable to more conventional local therapy, and is, I believe from experience, a considerable therapeutic advance. I prefer tablets to vaginal suppositories on technical and psychological grounds. Injections are rarely necessary. Occasionally unpleasant swelling of one or both breasts is a sequel, but is usually transitory.

\section{Infantile prematurity.}

Estrin by mouth, 500 units daily, may accelerate development. Thyroid is also useful for prematurity, grains I/ Io daily.

\section{PROGESTIN.}

This is the hormone of the corpus luteum, and is also termed progesterone. It is supplied, for intramuscular injection, in oily ampoules of I c.cm., containing I or 2 rabbit units. A rabbit unit is the equivalent of I $\mathrm{mg}$. The function of the corpus luteum is to convert the cestrin prepared endometrium to a secretory or pre-gestational phase, and also to maintain pregnancy.

\section{Menorrhagia.}

This may be due to a relative excess of œstrin and deficiency of progestin. Two rabbit units, repeated if necessary, may control the bleeding. (See also the discussion of menorrhagia under gonadotropic hormones.)

\section{Threatened or habitual miscarriage.}

Four rabbit units of progestin should be injected at the first sign of bleeding, and repeated daily if bleeding continues. Subsequently four rabbit units weekly are given. In a woman who has aborted by a previous pregnancy, such treatment should be begun a month before the time of expected abortion, and continued to the eighth month. It may be safe to discontinue injections after the usual time of an habitual abortion, e.g. fourth month.

It is assumed that more obvious causes, such as syphilis, have been excluded.

\section{MALE HORMONE.}

"Male" or comb-growth hormone is now available either as a urinary extract (hombreol) or as a synthetic preparation of testosterone propionate (Perandren, Neo-hombreol). The latter are concentrated preparations containing $5 \mathrm{mg}$. or 250 capon units per c.cm.

The hormone is responsible for secondary sexual characteristics, and for the proper development of the penis, prostate and seminal vesicles. The atrophy of these organs that follows castration can be corrected by this hormone. Experimentally, in large doses, it may inhibit testicular development by its depressing effect on pituitary gonadotropic function. This effect is not seen with clinical dosage, and, in fact, some observers find a stimulating effect.

\section{Hypogonadism.}

Whether this is part of an endocrine syndrome, or of no obvious origin, testosterone will repair those features that are due to lack of testicular secretion. The dosage may be 500 capon units twice weekly. (See also under gonadotropic hormone.) 


\section{Impotence.}

This condition is most frequently of psychological origin, but where due to hypogonadism appropriate hormone therapy (see above) is indicated.

\section{Prostatic symptoms.}

Since enlargement of the prostate occurs at an age when sexual potency is on the wane, it is reasonable to attribute the enlargement to deficiency of testicular hormone. Experimentally, œstrin is found to produce prostate enlargement, but testosterone, given at the same time, can prevent this action. This is the basis of a theory of prostatic enlargement.

The histological picture of prostatic enlargement in man, however, does not resemble that of experimental œstrin enlargement in lower animals, although the latter is similar to the spontaneously enlarged prostate of dogs.

In a man, with an adrenal tumour who excreted some 20,000 units of œstrin daily for several months, I could find no clinical evidence of prostatic enlargement, nor were there any urinary symptoms. This observation is perhaps in conflict with the œestrin theory of prostatic enlargement.

Several clinicians have tried the testosterone treatment of prostatic enlargement, and although many remain sceptical, there is some evidence that in large doses (20 mg. twice weekly) symptomatic improvement occurs. Some are of opinion that suggestion is sufficient to account for the benefit observed. Others have, perhaps paradoxically, obtained beneficial results from œstrin therapy.

I have tried the injection of 500 units of gonadotropic hormone (pregnyl three times weekly for three months, in a small series of cases (5), and have obtained no benefit. Nor was it possible, in these cases, to demonstrate any increase in the amount of comb-growth hormone in the urine, after the injections (Parkes): If the failure of testicular function in old age is comparable to the failure of ovarian function (see above) this is what might be expected, and one might postulate that the testis at this period of life is incapable of responding to gonadotropic stimulus. I believe, however, that one observer using 3,000 units of gonadotropic hormone per week was more favourably impressed with his results. Myxœdema in late middle life appears to be spontaneous, and not secondary to pituitary failure; there is in fact an excess of thyrotropic hormone in the serum, and Scowen has shown that the thyroid is unable to respond to injections of thyrotropic hormone. In fact, the circumstances appear to be quite comparable to the ovarian failure of the menopause, and one is therefore inclined to think that the failure of testicular function may be similar.

\section{ZINC PROTAMINE INSULIN.}

Ordinary insulin begins to act in twenty minutes, has a maximum effect in three hours, and has ceased to act within about eight hours. Its immediate action has obvious advantages, but efforts have been made to increase the duration of its action. Leyton used a suspension of insulin in castor oil. Clausen added minute quantities of adrenaline to insulin to retard absorption. Hagedorn succeeded in linking insulin to a natural protamine from fish sperm (insulin retard). Scott added traces of zinc to protamine insulin, thus producing still slower action.

This zinc protamine insulin constitutes a uniform suspension, and the nature of the physico-chemical union is not yet clearly defined. It is on the market in concentrations of 40 units and 80 units per c.cm. The zinc is present in only minute traces, I mg. in 500 units of insulin, and no evidence of zinc poisoning has been recorded. 
Zinc protamine insulin begins to act six hours after injection, has a maximum effect at twelve hours, and ceases to act after some twenty-eight hours. This time relationship has the advantage of making possible the control of sugar concentration, over a period of twenty-four hours, by a single injection instead of two or three.

It is usually given before breakfast (but in some patients an evening injection ensures a better balance). The fact that its action may not begin for some hours means that in some cases breakfast is followed by hyperglycæmia, and glycosuria. To obviate this a dose of ordinary insulin may be given at the same time. For exactitude, this should be given in a separate syringe, but even when given in the same syringe as the zinc protamine insulin (Lawrence and Archer), a variable but considerable proportion of the insulin retains its physico-chemical freedom, and rapid action. In actual practice, the method is often efficacious. Insulin retard (Hagedorn) has a time action curve intermediate between that of ordinary insulin and zinc protamine insulin.

Another advantage of zinc protamine insulin is that hypoglycamic reactions are less common. This is a great advantage in children when the blood sugar fluctuates wildly, severe hyperglycæmia alternating with hypoglycæmia. The hypoglycæmia that is precipitated by exercise is also a less frequent occurrence with zinc protamine insulin. When, however, hypoglycæmic attacks do occur they tend to be much more insidious in their onset, and therefore less easily detected by patient or doctor; further, they are more prolonged, and repeated administration of glucose over a number of hours may be necessary. It is therefore inadvisable to commence treatment with zinc protamine insulin except under medical supervision in hospital or nursing home.

It is assumed that with zinc protamine insulin, as with ordinary insulin, the value of the adoption of a diet containing some 150 to 200 grams of carbohydrate has been agreed upon. It has been shewn that such diets do not call for appreciably larger doses of insulin than the more unnatural low carbohydrate diets formerly used, and they tend to ensure proper development and a freedom from the complications of faulty fat metabolism.

If zinc protamine insulin is given before breakfast without ordinary insulin, it is advisable for the breakfast carbohydrate to be small, the lunch carbohydrate medium, and that of tea and supper larger, as this is the time when the maximum insulin effect will be in operation.

The use of zinc protamine insulin may have some other disadvantages. Thus, for mechanical reasons, its absorption and, therefore, its action may vary from day to day. Occasionally, a local allergic response may cause painful indurations at the site of injection, when ordinary insulin in the same patient has proved painless. However, the continued use of the zinc protamine insulin, or change to another proprietary preparation, may obviate this difficulty. Lawrence finds zinc protamine insulin a useful adjunct to ordinary insulin in the treatment of diabetic coma, but as I have found that a cumulative effect may be obtained even with ordinary insulin in diabetic coma (with a corresponding danger of severe hypoglycæmia), I would rather rely entirely on ordinary insulin in the coma and precoma phases of diabetes.

\section{The use of insulin in non-diabetic conditions.}

A proportion of patients suffering from wasting of indeterminate origin may benefit from insulin, Io units twice daily, given some twenty minutes before a high 
carbohydrate meal. Its exact mechanism is not certain, but its anabolic action is comparable to that seen in some diabetic children who become very fat under insulin therapy. It also increases appetite, probably by a vagus effect of the hypoglycæmia, producing hyperchlorhydria, and gastric motility, and may be useful therapy in anorexia. Insulin has also been used in the wasting of phthisis, but in this condition it should be remembered that even diabetic patients, with pulmonary tuberculosis, may undergo unaccountable phases of severe hypoglycæmia under ordinary insulin therapy.

Insulin has also been used in typhoid fever, and is said to have a favourable effect on the septic symptoms, fever, and course of the disease.

Recently, dementia pracox and other mental disorders have been treated by insulin hypoglycæmia. Good results will not. be obtained unless a severe degree of hypoglycæmia is obtained, preferably with coma. I have suggested that a partial explanation is the effect of the adrenaline secretion that accompanies hyperglycæmia, since beneficial effects are also obtained with the related sympathomimetic compounds, ephedrine and benzedrine. But there is probably also a direct action of the hypoglycæmic state on the brain, and multiple minute cerebral hæmorrhages have been observed in fatal cases.

\section{CORTIN.}

Extract of the suprarenal cortex is known by several trade names:-Eucortone, Eschatin, Cortin, etc. Its essential indication is Addison's disease of the suprarenal cortex. For a case of average severity the dosage is Io c.cm. daily, given intramuscularly. I use the latter route, now, even in crisis, as there is less liability to the anaphylactic reaction that may follow intravenous injection, and it is equally effective; nor do I find it necessary to give intravenous fluids in crisis. The use of salt will often permit a smaller maintenance dose of cortin. During infection, the daily dose of cortin should be doubled, as the demands of the body are increased, and the cortin relatively less effectual. I have not found the adrenotropic hormone of the pituitary of appreciable value in Addison's disease.

\section{Other uses of Cortin.}

As with many new remedies, cortin has been suggested as appropriate therapy for innumerable conditions. In only a few, however, is its use of value. In Simmonds' cachexia (anterior pituitary deficiency) there is secondary atrophy of the adrenal cortex, and cortin may be of some value as a partial substitution therapy. On similar grounds cortin may be of benefit in anorexia nervosa. In pregnancy, there is an increased demand upon the adrenal cortex, and an inadequate response is suggested by pigmentation, vomiting and asthenia-cortin may then be of value.

The blood chemistry and some clinical aspects of surgical shock suggest acute adrenal insufficiency, and cortin sometimes appears beneficial. Its value in shock following extensive burns is well testified to. In some acute infections and septicamias, hæmorrhage and necrosis occurs in the adrenal cortex, and the use of cortin may tide over a critical period. Similarly, it may be of value in diphtheria. There may be clinical evidence in favour of its use in infantile marasmus, gastroenteritis and prematurity. I have not found it of value in hyperthyroidism, but others have. Some consider it is useful in asthenia, associated with low blood sugar and low blood pressure, but not due to Addison's disease. I have found cortin to be quite useless in myasthenia gravis. 